\title{
Tumor de Wilms: Estudio de Centro Único de Los Andes Ecuatorianos. Serie de Casos con Seguimiento
}

\author{
Wilms' Tumor: Single Center Study From the \\ Ecuadorian Andes. Case Series With Follow-Up
}

\author{
Enmanuel Guerrero ${ }^{1,2,3}$; Raúl Alvarado ${ }^{1}$; Adriana Urdiales ${ }^{1}$; María José Orbe ${ }^{1}$; Orieta Navarrete \& $^{3}$ Carlos Manterola $^{3,4,5}$
}

GUERRERO, E.; ALVARADO, R.; URDIALES, A.; ORBE, M. J.; NAVARRETE, O. \& MANTEROLA, C. Tumor de Wilms: estudio de centro único de los andes ecuatorianos. Serie de casos con seguimiento. Int. J. Morphol., 38(1):208-214, 2020.

RESUMEN: El Tumor de Wilms (TW) es el tumor renal más frecuente entre los 1 y 5 años de edad. La evidencia existente respecto de aspectos clínicos, terapéuticos y de supervivencia (SV) del TW es escasa. El objetivo de este estudio fue determinar diferencias en la SV actuarial global (SVAG) y SV libre de enfermedad (SVLE) a 5 años en pacientes con TW tratados con quimioterapia neoadyuvante (QTNA) y cirugía inicial (CI). Serie de casos. Se incluyeron pacientes con TW de 11 meses y 13 años de edad, tratados en el Instituto del Cáncer SOLCA, Cuenca (1994-2019). Las variables resultado fueron SVAG y SVLE a 5 años. Otras variables de interés fueron: localización, estadio, histología, seguimiento y remisión completa (RC). Una vez concluidos sus tratamientos, los pacientes fueron sometidos a un seguimiento clínico. Se utilizó estadística descriptiva (medidas de tendencia central y dispersión) y analítica (Chi2, exacto de Fisher y corrección por continuidad). Se realizaron análisis de SV con curvas de Kaplan Meier y log-rank. Se reclutaron 36 pacientes (52,8 \% hombres), con una mediana de edad de 44 meses; 55,5\% de ellos tuvieron histología favorable. La localización y estadio más frecuente fue riñón izquierdo $(55,5 \%)$ y I (33,3\%) respectivamente. El 58,3\% fueron sometidos a CI y el 41,7 \% QTNA. Luego de aplicados los tratamientos 21 pacientes (58,3\%), alcanzaron RC. La SVAG y SVLE general a 5 años fue 72,0 \% y $69,0 \%$ respectivamente. Al comparar los subgrupos con QTNA y CI; se verificaron SVAG y SVLE a 5 años de 60,0 \% y 81,0 \% (p=0,118); y de $66,7 \%$ y $71,4 \%(p=0,536)$ respectivamente. La SVAG y SVLE verificadas son similares a las reportadas en otros estudios. No se evidenciaron diferencias de éstas con los tratamientos QTNA y CI.

PALABRAS ClAVE: Tumor de Wilms; Quimioterapia; Cirugía; Supervivencia.

\section{INTRODUCCIÓN}

El cáncer, constituye un grave problema de salud pública. Tendrá un incremento sostenido, proyectándose qué en 2030, será responsable del $70 \%$ del total de fallecimientos del mundo (Solomon, 2010); es decir, unos 12 millones de defunciones por año (Itriago et al., 2013).

Por su parte, los tumores renales (TR) en el ámbito pediátrico, representan el $7 \%$ de todas las neoplasias infantiles; y de estos, el Tumor de Wilms (TW) o nefroblastoma es el más frecuente, con una incidencia de 7,1 casos por cada millón de niños menores de 15 años (Chatenoud et al., 2014; AMS, 2017) (Fig. 1).

Según el VI informe de registro de tumores de la SOLCA, del año 2015, los TR tuvieron una tasa de inciden- cia de 4 casos por millón de niños en el mismo grupo de edad (Kohler et al., 2011). Afecta más a niñas que a niños con una relación 1 a 0,92 (AMS, 2017). En el $10 \%$ se asocia a alteraciones genéticas (genes WT1 y WT2) y sindromáticas.

Clínicamente se manifiesta como masa sólida palpable en el abdomen. A lo que se recomienda agregar pruebas diagnósticas generales, TAC (muy relevante para determinar estadios); y según el tipo histológico tumor, completar con biopsia o gammagrafía ósea (AbdelMaboud Ibrahim et $a l ., 2014)$. El estadio incluye 5 categorías y dependiendo de este, y la variedad histológica verificada, se indica quimioterapia, radioterapia o cirugía (PDQ Pediatric Treatment Editorial Board, 2002).

\footnotetext{
${ }^{1}$ Instituto del Cáncer SOLCA, Cuenca, Ecuador.

${ }^{2}$ Facultad de Medicina, Universidad de Cuenca, Cuenca, Ecuador.

${ }^{3}$ Programa de Doctorado en Ciencias Médicas, Universidad de La Frontera, Temuco, Chile.

${ }^{4}$ Departamento de Cirugía, Universidad de La Frontera, Temuco, Chile.

${ }^{5}$ Centro de Estudios Morfológicos y Quirúrgicos (CEMyQ), Universidad de La Frontera, Temuco, Chile.
} 


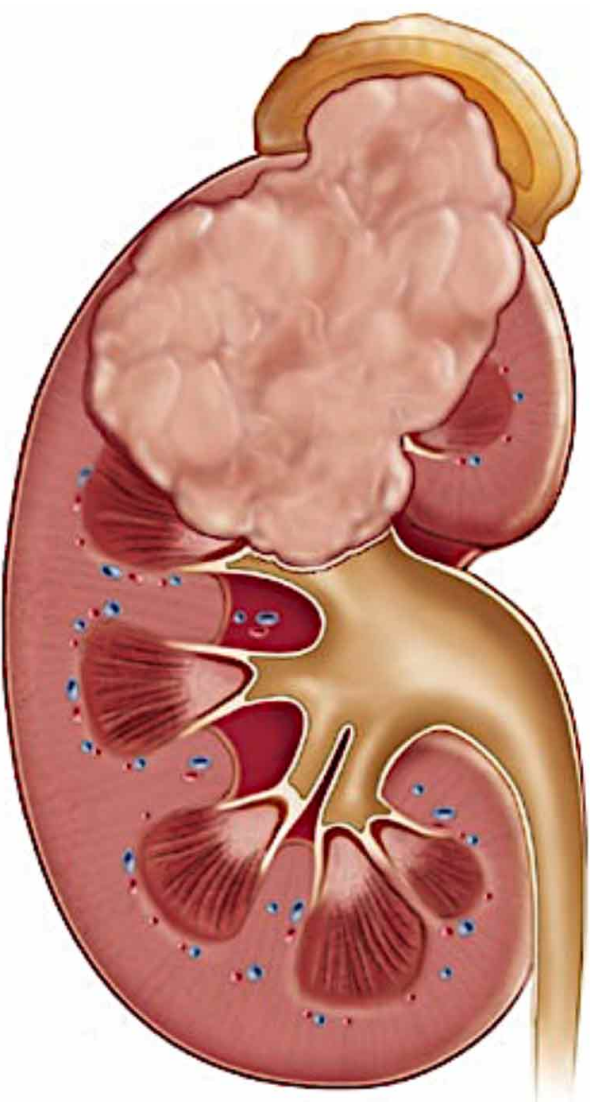

Fig. 1. Aspectos morfológicos macro y microscópico del TW.
La supervivencia actuarial global (SVAG) y la supervivencia libre de enfermedad (SVLE) a 5 años en pacientes con TW es de $100 \%$ a 73,6 \%; y de 95,4\% a 50,0\%, según estadios (I a V) respectivamente (Guruprasad et al., 2013; Doganis et al., 2019).

El objetivo de este estudio fue determinar diferencias en la SV actuarial global (SVAG) y SV libre de enfermedad (SVLE) a 5 años en pacientes con TW tratados con quimioterapia neoadyuvante (QTNA) y cirugía inicial (CI).

\section{MATERIAL Y MÉTODO}

Este artículo fue escrito siguiendo la iniciativa MInCir para el reporte de estudios observacionales descriptivos (Manterola \& Otzen, 2017).

Diseño: Serie de casos con seguimiento.

Centro: El estudio fue realizado en el Instituto del Cáncer SOLCA-Cuenca, en el período enero de 1994 y abril de 2019 (25 años).
Participantes: Se incluyeron todos los pacientes con diagnóstico histopatológico de TW, de entre 11 meses y 13 años de edad; en el período e Institución antes señalados, tratados con QTNA y CI. Fueron excluidos aquellos casos con información incompleta.

Tamaño de muestra: Se trabajó con la totalidad del universo de pacientes con TW, sometidos a los tratamientos antes mencionados en el período e institución señalada. Por ende, no se realizó estimación de tamaño de la muestra.

Variables: Las variables resultado fueron SVAG y SVLE (general y según tratamiento con QTNA y CI), medida en meses posterior a la aplicación de las terapias antes mencionadas. Otras variables de interés fueron: biodemográficas, presencia de hematuria, estadio TNM según la American Cancer Society (AMS, 2017), histopatología, volumen tumoral, niveles de deshidrogenasa láctica (DHL), mortalidad asociada a los tratamientos y recurrencia. En aquellos pacientes en los que el estadio clínico no estaba claramente definido, se utilizaron las directrices actuales recomendadas por la NWTS (National Wilms Tumor Study). 
Seguimiento: El seguimiento mínimo y máximo fue de 4 y 256 meses (mediana de 77 meses). Este, se realizó mediante el estudio de las historias clínicas, base de datos institucional y certificados de defunción obtenidos de la página Web del registro civil.

Estadísticas: Utilizando el paquete estadístico SSPS 23.0, se realizó un análisis exploratorio de los datos. Se aplicó estadística descriptiva con cálculo de porcentajes, medidas de tendencia central y de dispersión. Se utilizó estadística analítica para comparación de promedios, con t-test; y Chi2 de Pearson, exacto de Fisher y corrección por continuidad, para comparación de variables categóricas. Finalmente se realizaron curvas de SV de Kaplan Meier, y prueba de logrank para comparación de SV con los tratamientos QTNA y CI.

Principios éticos: Todos los padres o tutores de los pacientes firmaron un consentimiento informado y sus identidades se mantuvieron ocultas luego de que fueron codificados cada uno de ellos.

\section{RESULTADOS}

En el período estudiado, se trataron 45 pacientes con diagnóstico de TW. De estos, 9 fueron excluidos por no cumplir con los criterios de selección. La mediana de edad de los 36 pacientes al momento del diagnóstico fue de 44 meses (11-148 meses). El 52,8\% (19 pacientes), eran de sexo masculino. Las características clínicas y patológicas se detallan en la Tabla I.

La mediana del volumen tumoral reportados en la TAC y el estudio de las piezas quirúrgicas fue de 952 cc (180-2904 cc); y de la DHL fue de 772 U/L (184-3104 U/L).

Las características de la quimioterapia recibida por los pacientes según estadio y condición se detallan en la Tabla II.

De los 21 pacientes que alcanzaron la RC, tres (14\%) tuvieron recaída (mediana de 3 meses). Del total de pacientes, cinco (13,9\%), fallecieron (mediana de 10 meses); tres eran del grupo de CI y dos de QTNA.

Las complicaciones observadas con la quimioterapia se enumeran en la Tabla III.

Como se observa en la Tabla IV, solo 16 recibieron radioterapia como parte de su tratamiento. De los 20 pacientes no irradiados, 11 pertenecían a estadios bajos ( 8 al
Tabla I. Características patológicas, clínicas y terapéuticas de los pacientes en estudio. $(\mathrm{N}=36)$.

\begin{tabular}{|c|c|c|}
\hline Variables & $\mathrm{N}^{\circ}$ casos & $\%$ \\
\hline \multicolumn{3}{|l|}{ Subtipo histológico } \\
\hline Desconocido & 14 & 38,9 \\
\hline Blastematoso & 8 & 22,2 \\
\hline Mixto & 6 & 16,7 \\
\hline Otros & 8 & 22,2 \\
\hline \multicolumn{3}{|l|}{ Histología } \\
\hline Favorable & 20 & 55,5 \\
\hline No favorable & 2 & 5,6 \\
\hline Desconocido & 14 & 38,9 \\
\hline \multicolumn{3}{|l|}{ Hematuria al diagnóstico } \\
\hline Sí & 9 & 25,0 \\
\hline No & 24 & 66,7 \\
\hline Desconocido & 3 & 8,3 \\
\hline \multicolumn{3}{|l|}{ Estadio } \\
\hline I & 12 & 33,3 \\
\hline II & 5 & 13,9 \\
\hline III & 10 & 27,8 \\
\hline IV & 8 & 22,2 \\
\hline $\mathrm{V}$ & 1 & 2,8 \\
\hline \multicolumn{3}{|l|}{ Localización } \\
\hline Izquierda & 20 & 55,5 \\
\hline Derecha & 15 & 41,7 \\
\hline Bilateral & 1 & 2,8 \\
\hline \multicolumn{3}{|l|}{ Radioterapia } \\
\hline Sí & 16 & 44,4 \\
\hline No & 20 & 55,6 \\
\hline \multicolumn{3}{|l|}{ Tratamiento Inicial } \\
\hline QTNA & 15 & 41,7 \\
\hline $\mathrm{CI}$ & 21 & 58,3 \\
\hline \multicolumn{3}{|l|}{ CI y CP QTNA } \\
\hline NI & 15 & 41,7 \\
\hline ND & 10 & 27,8 \\
\hline $\mathrm{ND}+$ otro procedimiento & 4 & 11,1 \\
\hline $\mathrm{NI}+$ otro procedimiento & 4 & 11,1 \\
\hline No se realizó cirugía & 3 & 8,3 \\
\hline \multicolumn{3}{|l|}{ Complicaciones post cirugía } \\
\hline No presentó & 30 & 83,4 \\
\hline Neumotórax & 2 & 5,6 \\
\hline Bridas intestinales & 1 & 2.7 \\
\hline No se realizó cirugía & 3 & 8.3 \\
\hline
\end{tabular}

QTNA: Quimioterapia neoadyuvante CI: Cirugía inicial.CP QTNA: Cirugía Post quimioterapia neoadyuvante ND: Nefrectomía derecha. NI: Nefrectomía izquierda.

estadio I y 3 al estadio II) y eran de histología favorable; 5 abandonaron el tratamiento; 3 de estadios bajos e histología desconocida y uno falleció. La SVAG y SVLE fue de $72 \%$ y $69 \%$ respectivamente (Figs. 2 y 3). La SVAG verificada en pacientes sometidos a QTNA y CI fue $60,0 \%$ y $81,0 \%$ respectivamente ( $p>0,05)$. (Fig. 4). La SVLE observada en pacientes sometidos a QTNA y CI fue $66,7 \%$ y $71,4 \%$ respectivamente ( $p>0,05)$. (Fig. 5). 
GUERRERO, E.; ALVARADO, R.; URDIALES, A.; ORBE, M. J.; NAVARRETE, O. \& MANTEROLA, C. Tumor de Wilms: estudio de centro único de los andes ecuatorianos. Serie de casos con seguimiento. Int. J. Morphol., 38(1):208-214, 2020.

Tabla II. Quimioterapia, estadio y condición luego del tratamiento administrado a los pacientes en estudio. ( $\mathrm{N}=36)$

\begin{tabular}{lccccccc}
\hline & Estadio & \multicolumn{5}{c}{ Condición post- tratamiento } \\
& & RC & Progr. & Abandono & Muerte & En tto. & Total \\
\hline \multirow{2}{*}{ ACT/VCR/DOXO } & I & 1 & - & - & - & - & 1 \\
(24 semanas) & II & - & 1 & - & - & - & 1 \\
& III & 8 & - & - & - & - & 8 \\
& IV & 1 & 1 & 1 & 3 & - & 6 \\
ACT/VCR & I & 7 & - & 1 & 1 & 1 & 10 \\
(18 semanas) & II & 2 & - & - & 1 & - & 3 \\
& III & - & - & 1 & - & - & 1 \\
& IV & - & - & 1 & - & - & 1 \\
ACT/VCR/DOXO/CICLO & V & - & - & 1 & - & - & 1 \\
(24 semanas) & I & - & - & 1 & - & - & 1 \\
& II & - & - & 1 & - & - & 1 \\
Total & III & 1 & - & - & - & - & 1 \\
\hline ACT: Actir & IV & 1 & - & - & - & - & 1 \\
\hline
\end{tabular}

ACT: Actinomicina D 1,35 mg/m²/dosis. VCR: Vincristina 1,5-2mg/m²/dosis. DOXO: Doxorrubicina 30-45mg/m2/dosis. CICLO: Ciclofosfamida 440-500mg/m2/día. RC: Remisión completa. RP: Remisión parcial. Prog.: Progresión. En tto.: En tratamiento.

Tabla III. Complicaciones post-quimioterapia en los pacientes en estudio. $(\mathrm{N}=31)$.

\begin{tabular}{lcc}
\hline Complica ciones & Frecuencia & $\%$ \\
\hline Alopecía & 31 & 100,0 \\
Hepatopatía & 25 & 80,6 \\
Nausea/vómito & 25 & 80,6 \\
Anemia & 24 & 77,4 \\
Neutropenia & 19 & 61,2 \\
Dolor abdominal & 16 & 51,6 \\
Trombocitopenia & 14 & 45,1 \\
Diarrea & 12 & 38,7 \\
Estreñimiento & 10 & 32,2 \\
Otras & 18 & 58,0 \\
\hline
\end{tabular}

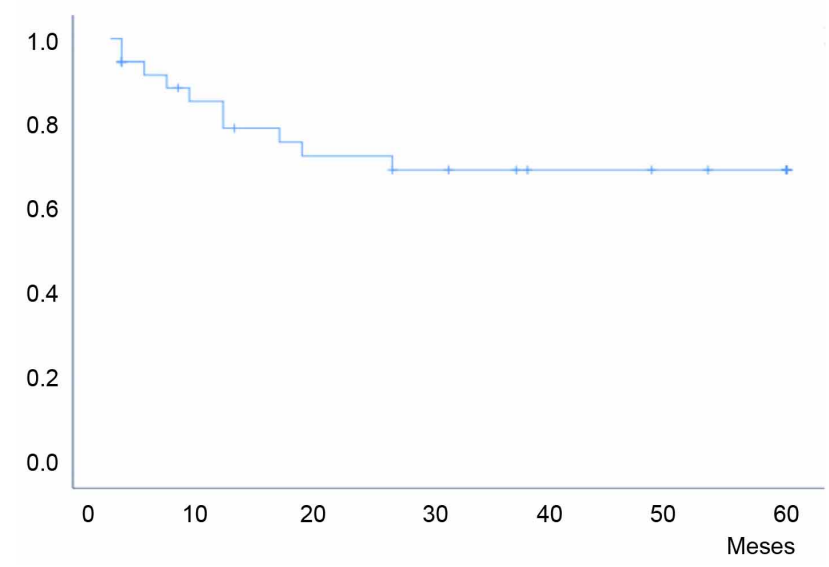

Fig. 2. SVGA a 5 años para los pacientes con TW en Estudio.
Tabla IV. Radioterapia administrada y localización en los pacientes en estudio. $(\mathrm{N}=16)$.

\begin{tabular}{lcc}
\hline Localización & Frecuencia & $\%$ \\
\hline Fosa renal & 9 & 56,2 \\
Hemiabdomen & 3 & 18,8 \\
Hemiabdomen y pulmón & 2 & 12,6 \\
Pulmón & 1 & 6,2 \\
Fosa renal y cráneo & 1 & 6,2
\end{tabular}

Dosis (Gy): promedio de 18,5 $\pm 12,2$ (11 a 49). Mediana de 10,8.

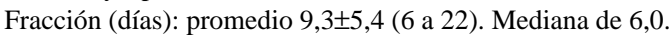

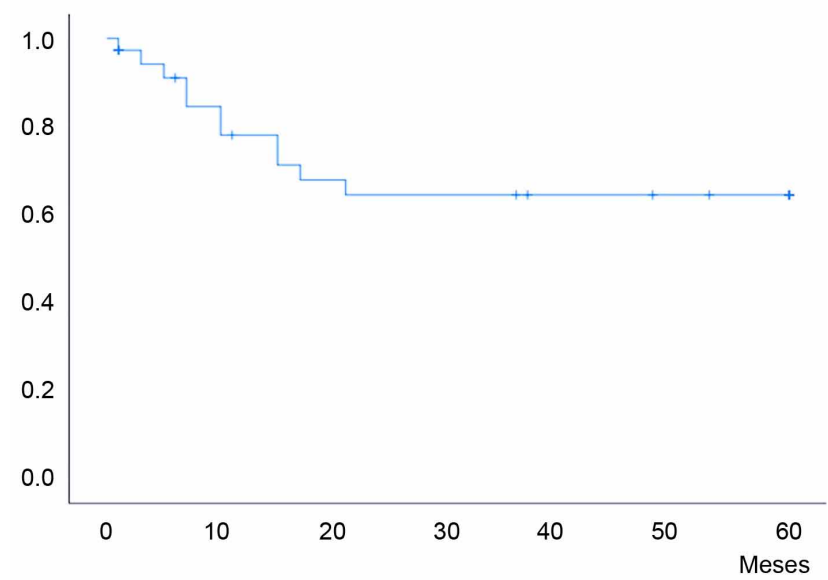

Fig. 3. SVLE a 5 años para los pacientes con TW en estudio. 


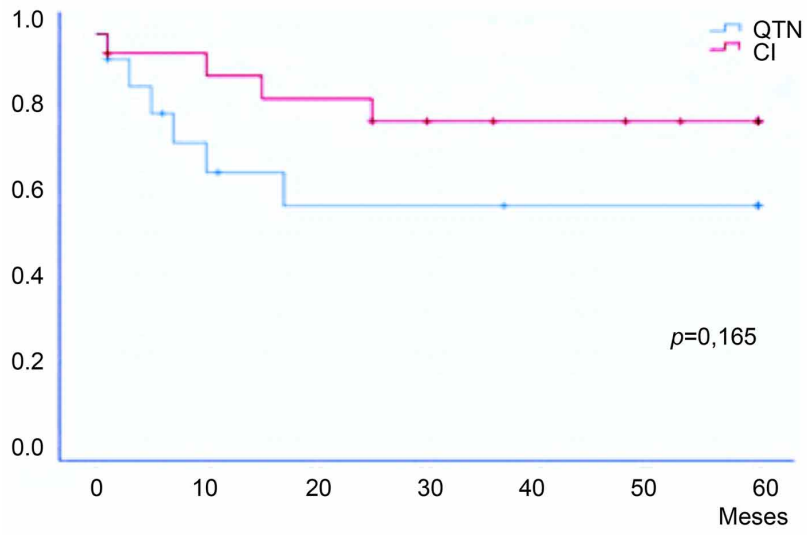

Fig. 4. SVGA a 5 años para los pacientes con TW en estudio tratados con QTNA y CI.

\section{DISCUSIÓN}

Este es el mayor estudio de TR, de un único centro a nivel del Ecuador, que realizó este tipo de revisión, en este grupo de edad. Se demostró que las características clínicas y patológicas son similares a las reportadas en otros estudios. Los estadios clínicos avanzados son más frecuentes. Los esquemas de tratamiento (quimioterapia, radioterapia y cirugía) aplicados a los pacientes dependen de su estadio e histología y se basan en la mejor evidencia disponible dados por el COG y SIOP.

La novedad de la propuesta es que se trata de una casuística unicéntrica y regional, que concentra la totalidad de pacientes oncológicos tanto públicos como privados del sur del país; con resultados comparables con diferentes series provenientes de otros países, en términos de SVAG y SVLE.

Entre las limitaciones del estudio, cabe señalar que se trata de una serie pequeña; y que, en los últimos casos, el seguimiento es aún demasiado corto.

El TW es el tumor renal más frecuente en la edad pediátrica (5-8\%). La edad promedio del diagnóstico es a los 44 meses, que coincide con la mediana de edad de este estudio (PDQ Pediatric Treatment Editorial Board).

Es más frecuente en niñas e involucra por igual a ambos riñones (PDQ Pediatric Treatment Editorial Board; Lamb et al., 2017; Tan et al., 2018). En este estudio, estos resultados fueron diferentes (niños 52,8 \% y lado izquierdo $55,5 \%$ ), y pudo deberse al tamaño y azar de la muestra.

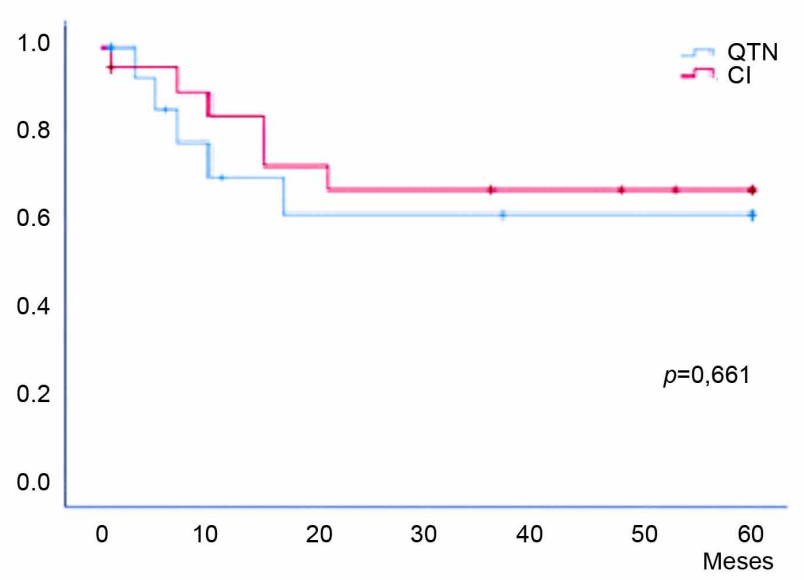

Fig. 5. SVLE a 5 años para los pacientes con TW en estudio tratados con QTNA y CI.

La palpación de una masa tumoral es el signo clínico más común. No existió un reporte confiable de este signo en las historias clínicas de los pacientes por lo que no fue considerado este signo dentro de las variables de estudio.

Puede ser de histología favorable (blastemal, estromal y epitelial con ó sin anaplasia focal) y no favorable (anaplasia difusa). El Children's Oncology Group (COG) y la Sociedad Internacional de Oncología Pediátrica (SIOP), también incluyen dentro de la histología no favorable al tumor Rabdoide del riñón y al tumor de Células Claras (Lamb et al.).

La anaplasia difusa y la histología no favorable muestran malos resultados en la supervivencia y mayor mortalidad, porque están relacionados a mutaciones del gen P53 (gen supresor de tumores) (SIOP 2015 Scientific Programme + Index, 2015; Vujanic et al., 2018).

Según el NWTS, cuando el TW se limita al riñón (cápsula intacta, sin afectación vascular) y se reseca completamente se considera estadio I. En el estadio II, el tumor se reseca completamente, pero existe infiltración capsular y vascular. El estadio III incluye residuo tumoral posquirúrgico macroscópico, ganglios linfáticos abdomino-pélvicos positivos, implantes peritoneales, extensión a la vena cava, ruptura o biopsia tumoral (Irtan et al., 2015; Tan et al.).

En países desarrollados, los estadios I, II, III, y IV pueden presentarse en el $43 \%, 20 \%, 21 \%$ y $11 \%$ de los 
casos respectivamente (PDQ Pediatric Treatment Editorial Board), con una alta SVLE a los 5 años (EE.UU. $90 \%$, Italia $86 \%$, Alemania $84 \%$ y Reino Unido $82 \%$ ), mientras que en países de bajos ingresos (África Subsahariana); la SVLE oscila entre 11 y $46 \%$ (Njuguna et al., 2017). Este estudio fue realizado en un país de recursos económicos medios, por ende, los estadios III y IV fueron mas frecuentes $(50 \%)$ y consecuentemente la SVLE fue menor $(69,4$ $\%)$, lo que da a entender que estos resultados pueden depender del nivel de desarrollo de un país y de los recursos económicos destinados a la salud de sus habitantes.

Igual explicación tendrían la DHL (4 veces más alto del rango normal) y el volumen tumoral (mediana 952cc), que por ejemplo, al comparar este último con el volumen tumoral de los pacientes con TW de Alemania (572cc) y Reino Unido ( $381 \mathrm{cc})$, estos fueron menos voluminosos (SIOP 2015 Scientific Programme + Index; Zhou et al., 2019).

Dependiendo del estadio e histología la quimioterapia puede cambiar. En los estadios I y II se recomienda Vincristina y Actinomicina D. En el estadio III se utiliza además doxorrubicina y ciclofosfamida. Para el estadio IV se incluye etopósido. El tiempo varía entre 18 y 24 semanas (PDQ Pediatric Treatment Editorial Board; Dix et al., 2018). Los pacientes de este estudio sometidos a quimioterapia, presentaron complicaciones secundarias típicas y transitorias descritas en la bibliografía (Velásquez et al., 2016; de Francisco et al., 2019).

Todos los pacientes, fueron analizados en sesión médica donde se realizó esta investigación y el tratamiento fue aplicado siguiendo las guías recomendadas por el COG (CI seguido de quimioterapia) y la SIOP (QTNA seguido de cirugía y quimioterapia). En caso de ser necesario ambos grupos utilizan radioterapia con similares supervivencias. El uso de QTNA modifica las características patológicas del TW, haciéndole en algunos casos imposible determinar el tipo histológico (Van den Heuvel-Eibrink et al., 2015; Junjun et al., 2015). Ocho $(57,1 \%)$ de los 14 pacientes con histología desconocida, habían recibido QTNA.

El 41,7 \% (15 pacientes) recibieron de 3 a 6 semanas de QTNA, resultado similar al reportado por la Asociación Italiana de Hemato-Oncología Pediátrica (AIEOP-TW2003), quienes también dieron QTNA al $42 \%$ de sus pacientes, evidenciándose que este tratamiento puede ser aplicado correctamente en cualquier área geográfica (Spreafico et al., 2017).

La radioterapia se administra sobre todo a los pacientes con estadios III y IV. La dosis varía según la edad, zona a irradiar, estadio y respuesta quimioterapéutica. Esta oscila entre 10,8 a 21 Gy y cuando se cumple en menos de 14 días se relaciona con una mejor supervivencia (Stokes $e t$ al., 2018).

La cirugía es el tratamiento de elección. Está contraindicada cuando el tumor infiltra la vena Cava, o cuando sobrepasa la línea media del abdomen (por el riesgo de que se rompa y contamine la cavidad abdominal) (Ezomike et al., 2018). A 21 pacientes $(58,3 \%)$ se les realizó CI, y cuando se sumaron los pacientes con QTNA fueron intervenidos 33 pacientes $(91,67 \%)$. En ningún paciente hubo ruptura tumoral por causa quirúrgica (a pesar del gran volumen tumoral) y las complicaciones post quirúrgicas fueron en 3 pacientes.

Los estadios avanzados se asocian a bajas supervivencias (Njuguna et al.; Vujanic et al.). Tanto la SVAG $(\mathrm{p}=0,118)$ y la SVLE $(\mathrm{p}=0,536)$ fueron levemente mejores en los pacientes con CI que en aquellos con QTNA, debido a que en el grupo que fueron a CI el 71,4\% pertenecieron a los estadios I y II y en el grupo de QTNA el $12,5 \%$. La SVAG fue similar a la reportada en una reciente revisión sistemática (Han et al., 2018).

A modo de conclusión, se puede señalar que los resultados verificados en esta serie, en términos de SVAG y SVLE a 5 años en pacientes con TW, son comparables a series de centros de derivación nacionales e internacionales en los que no se ha aplicado terapias neoadyuvantes.

GUERRERO, E.; ALVARADO, R.; URDIALES, A.; ORBE, M. J.; NAVARRETE, O. \& MANTEROLA, C. Wilms' tumor: single center study from the ecuadorian andes. Case series with follow-up. Int. J. Morphol., 38(1):208-214, 2020.

SUMMARY: Wilms tumor (WT) is the most common pediatric kidney tumor between 1 and 5 years of age. The existing evidence regarding clinical, therapeutic and survival (SV) aspects of TW is scarce. The aim of this study was to determine differences in 5-year overall survival (OS) and 5-year disease-free survival (DFS), in patients treated by WT with neoadjuvant chemotherapy (NACT) and initial surgery (IS). Case series. Patients with TW between 11 months and 13 years of age, treated at SOLCA Cancer Institute, Cuenca, Ecuador (1994-2019) were included. The outcome variables were OS and DFS. Once their treatments were completed, patients were followed clinically. Descriptive (measures of central tendency and dispersion) and analytical (Chi2, Fisher's exact and continuity correction) statistics were applied. SV analysis with Kaplan Meier curves and log-rank were performed. 36 patients (52.8\% men), with a median age of 44 months; $55.5 \%$ of which had favorable histology were recruited. The most frequent location and stage was left kidney $(55.5 \%)$ and I $(33.3 \%)$ respectively. $58.3 \%$ underwent IC and $41.7 \%$ QTNA. After treatments, 21 
patients $(58.3 \%)$ achieved complete remission. General OS and DFS were $72.0 \%$ and $69.0 \%$ respectively. When comparing subgroups with QTNA and CI. When comparing the subgroups with QTNA and CI, OS and DFS of $60.0 \%$ and $81.0 \%$ were verified $(\mathrm{p}=0.118)$; and of $66.7 \%$ and $71.4 \%(\mathrm{p}=0.536)$ respectively. General OS and DFS observed are similar to those reported in other studies. No differences were evidenced with QTNA and CI treatments.

KEY WORDS: "Wilms Tumor"[Mesh]; "Kidney Neoplasms"[Mesh]; "Chemotherapy"[Mesh]; "Survival Analysis"[Mesh]; "Disease-Free Survival"[Mesh].

\section{REFERENCIAS BIBLIOGRÁFICAS}

AbdelMaboud Ibrahim, N. M.; Elsaid, H. H. \& Aly aboubeih, E. The accuracy of MDCT for staging of Wilms' tumor. Egypt. J. Radiol. Nuclear Med., 45(2):575-81, 2014.

American Cancer Society (AMS). American Joint Committee on Cancer Bone AJCC Cancer Staging Manual. $8^{\text {th }}$ ed. New York, Springer, 2017. pp.471.

Chatenoud, L.; Bertuccio, P.; Bosetti, C.; Malvezzi, M.; Levi, F.; Negri, E. \& La Vecchia, C. Trends in mortality from major cancers in the Americas: 1980-2010. Ann. Oncol., 25(9):1843-53, 2014.

de Francisco, L. M.; Macía, M.; Alonso, F.; García, P.; Gutierrez, E.; Quintana, L. F.; Quiroga, B. \& Torregrosa, I. Onco-Nefrología: cáncer, quimioterapia y riñón. Nefrología, 39(5):455-562, 2019.

Dix, D. B.; Seibel, N. L.; Chi, Y. Y.; Khanna, G.; Gratias, E.; Anderson, J. R.; Mullen, E. A.; Geller, J. I.; Kalapurakal, J. A.; Paulino, A. C.; et al. Treatment of stage IV favorable histology Wilms tumor with lung metastases: a report from the children's oncology group AREN0533 Study. J. Clin. Oncol., 36(16):1564-70, 2018.

Doganis, D.; Zborovskaya, A.; Trojanowski, M.; Zagar, T.; Bouka, P.; Baka, M.; Moschovi, M.; Polychronopoulou, S.; Papakonstantinou, E.; Tragiannidis, A.; et al. Wilms tumour event-free and overall survival in Southern and Eastern Europe: Pooled analyses of clinical data from four childhood cancer registries (1999-2017). Eur. J. Cancer., 115:37-46, 2019.

Ezomike, U. O.; Modekwe, V. I. \& Ekenze, S. O. Paediatric nephrectomy: Patterns, indications and outcome in a developing country. Malawi Med. J., 30(2):94-8, 2018.

Guruprasad, B.; Rohan, B.; Kavitha, S.; Madhumathi, D. S.; Lokanath, D. \& Appaji, L. Wilms' tumor: single centre retrospective study from South India. Indian J Surg Oncol., 4(3):301-4, 2013.

Han, Q.; Li, K.; Dong, K.; Xiao, X.; Yao, W. \& Liu, G. Clinical features, treatment, and outcomes of bilateral Wilms' tumor: A systematic review and meta-analysis. J. Pediatr. Surg., 53(12):2465-9, 2018.

Irtan, S.; Jitlal, M.; Bate, J.; Powis, M.; Vujanic, G.; Kelsey, A.; Walker, J.; Mitchell, C.; Machin, D.; Pritchard-Jones, K.; et al. Risk factors for local recurrence in Wilms tumour and the potential influence of biopsy - the United Kingdom experience. Eur. J. Cancer, 51(2):225-32, 2015.

Junjun, J.; Xuelian, Z.; Dhruba, K.; Haiyang, X.; Lin, Z. \& Shusen, Z. Efficacy of preoperative chemotherapy in treatment of children with Wilms' tumor: a meta-analysis. Iran. J. Pediatr., 25(2):e366, 2015.

Kohler, B. A.; Ward, E.; McCarthy, B. J.; Schymura, M. J.; Ries, L. A.; Eheman, C.; Jemal, A.; Anderson, R. N.; Ajani, U. A. \& Edwards, B. K. Annual report to the nation on the status of cancer, 1975-2007, featuring tumors of the brain and other nervous system. J. Natl. Cancer Inst., 103(9):714-36, 2011.
Lamb, M. G.; Aldrink, J. H.; O’Brien, S. H.; Yin, H.; Arnold, M. A. \& Ranalli, M. A. Renal tumors in children younger than 12 months of age: a 65-year single institution review. J. Pediatr. Hematol. Oncol., 39(2):103-7, 2017.

Manterola, C. \& Otzen, T. Checklist for reporting results using observational descriptive studies as research designs. The MInCir initiative. Int. J. Morphol., 35(1):72-6, 2017.

Njuguna, F.; Martijn, H. A.; Kuremu, R. T.; Saula, P.; Kirtika, P.; Olbara, G.; Langat, S.; Martin, S.; Skiles, J.; Vik, T.; et al. Wilms Tumor Treatment Outcomes: Perspectives From a Low-Income Setting. J. Glob. Oncol., 3(5):555-62, 2017.

PDQ Pediatric Treatment Editorial Board, P. P. T. E. Wilms Tumor and Other Childhood Kidney Tumors Treatment (PDQ $\left.{ }^{\circledR}\right)$ : Health Professional Version. PDQ Cancer Information Summaries. Bethesda, National Cancer Institute (US), 2002.

SIOP 2015 Scientific Programme + Index. Pediatr. Blood Cancer, 62(S4):S143-S418, 2015.

Spreafico, F.; Biasoni, D.; Lo Vullo, S.; Gandola, L.; D'Angelo, P.; Terenziani, M.; Bianchi, M.; Provenzi, M.; Indolfi, P.; Pession, A.; et al. Results of the Third AIEOP Cooperative Protocol on Wilms Tumor (TW2003) and Related Considerations. J. Urol., 198(5):1138-45, 2017.

Stokes, C. L.; Stokes, W. A.; Kalapurakal, J. A.; Paulino, A. C.; Cost, N. G.; Cost, C. R.; Garrington, T. P.; Greffe, B. S.; Roach, J. P.; Bruny, J. L.; et al. K. Timing of Radiation Therapy in Pediatric Wilms Tumor: A Report From the National Cancer Database. Int. J. Radiat. Oncol. Biol. Phys., 101(2):453-61, 2018.

Tan, X. H.; Zhang, D. Y.; Liu, X.; Lin, T.; He, D. W.; Li, X. L. \& Wei, G. H. Retrospective analysis to determine outcomes of patients with bilateral Wilms tumor undergoing nephron sparing surgery: 15-year tertiary single-institution experience. Pediatr. Surg. Int., 34(4):427-33, 2018.

van den Heuvel-Eibrink, M. M.; van Tinteren, H.; Bergeron, C.; Coulomb-L'Hermine, A.; de Camargo, B.; Leuschner, I.; Sandstedt, B.; Acha, T.; Godzinski, J.; Oldenburger, F.; et. al. Outcome of localised blastemal-type Wilms tumour patients treated according to intensified treatment in the SIOP WT 2001 protocol, a report of the SIOP Renal Tumour Study Group (SIOP-RTSG). Eur. J. Cancer, 51(4):498-506, 2015.

Velásquez, C. A.; González, M.; Berrouet, M. C. \& Jaramillo, N. Cardiotoxicidad inducida por la quimioterapia desde las bases moleculares hasta la perspectiva clínica. Rev. Colomb. Cardiol., 23(2):104-11, 2016.

Vujanic, G. M.; Gessler, M.; Ooms, A. H. A. G.; Collini, P.; Coulombl'Hermine, A.; D'Hooghe, E.; de Krijger, R. R.; Perotti, D.; Pritchard-Jones, K.; Vokuhl, C.; et al. The UMBRELLA SIOPRTSG 2016 Wilms tumour pathology and molecular biology protocol. Nat. Rev. Urol., 15(11):693-701, 2018.

Zhou, S. N.; Chen, Q. C.; Jiang, M. W.; Jiang, X. M.; Peng, R. B.; Han, Q. X.; Huang, C.; Zhao, C. X. \& Li, J. P. Effects of light intensity on growth, survival, metabolism and related enzyme activities of Sepia pharaonis. Ying Yong Sheng Tai Xue Bao, 30(6):2072-8, 2019.

\section{Dirección para correspondencia:}

Dr. Carlos Manterola

Departamento de Cirugía y CEMyQ

Universidad de La Frontera

Temuco - CHILE

Email: carlos.manterola@ufrontera.cl

Recibido: 03-08-2019

Aceptado: 06-09-2019 